\title{
ON ALLOCATION OF EXCESS OF LOSS PREMIUMS
}

\author{
BY BJøRN SUNDT \\ The Wyatt Company \& University of Oslo
}

\begin{abstract}
In the present paper we study the question of how to allocate the reinsurance premium between the sub-portfolios when an excess of loss treaty is to be shared between several sub-portfolios. Several allocation schemes based on the expected value principle and the standard deviation principle are suggested. The calculations are relatively simple with unlimited free reinstatements. However, with limited and/or paid reinstatements the situation becomes rather tricky, and we therefore suggest a simulation scheme.
\end{abstract}

\section{KEYWORDS}

Excess of loss reinsurance; reinstatements; allocation; stochastic simulations.

\section{INTRODUCTION}

An insurance portfolio covered by an excess of loss treaty can sometimes in a natural way be split into several sub-portfolios. These sub-portfolios can for instance be related to different lines of insurance (e.g. fire and accident), different geographical areas, different subsidiaries of an insurance company, etc. Sometimes it is desirable to allocate the reinsurance premium between the sub-portfolios, e.g. for accounting. In the present paper we shall discuss such allocation.

In Section 2 we make some general assumptions about the probabilistic model and the excess of loss treaty and introduce some quantities that we shall make use of later in the paper. For free reinstatements the allocation problem is much simpler than in the general case, and Section 3 is devoted to this special case. We discuss allocation both by the expected value principle and the standard deviation principle. Without the assumption about free reinstatements, we return to the expected value principle in Section 4 and the standard deviation principle in Section 5. Unfortunately, in the general case it can be rather complicated to estimate the moments appearing in our premium formulae, and in Section 6 we therefore suggest a simulation procedure. In Section 7 we briefly discuss some possible generalisations of our model.

\section{MODEL ASSUMPTIONS}

We consider an insurance portfolio that can be split into $S$ sub-portfolios. The portfolio is to be reinsured with an excess of loss treaty for the layer $m$ xs $l$ with 
$K$ reinstatements. The $k$ th reinstatement is at $100 c_{k} \%$, and the initial premium is $P$. For definitions of the reinsurance terminology applied in this paper, we refer to SUNDT (1991b).

Let $N$ be the number of claims incurred in the portfolio during the reinsurance period and $Y_{i}$ the amount of the $i$ th of these claims. For simplicity we assume that $Y_{i}$ is known immediately when the claim occurs. We introduce the part of $Y_{i}$ that falls within the layer $m \times s l$, that is,

$$
Z_{i}=\min \left(\max \left(Y_{i}-l, 0\right), m\right) .
$$

It is assumed that each claim falls totally within one sub-portfolio. Let $H_{i}$ denote the sub-portfolio of the $i$ th claim. We assume that the pairs $\left(H_{1}, Y_{1}\right),\left(H_{2}, Y_{2}\right), \ldots$ are mutually independent and identically distributed and independent of $N$. Let

$$
w_{h}=\operatorname{Pr}(H=h) . \quad(h=1, \ldots, S)
$$

(We drop indices when they do not contain any information.) We introduce

$$
X=\sum_{i=1}^{N} Z_{i}
$$

being the total recoveries if there were an infinite number of reinstatements, and the part of these incurred by sub-portfolio $h$

$$
X_{h}=\sum_{i=1}^{N} I\left(H_{i}=h\right) Z_{i} \quad(h=1, \ldots, S)
$$

with $I$ denoting the indicator function $(I(A)=1$ if the event $A$ occurs and $I(A)=0$ else $)$. We also introduce the corresponding relative quantities

$$
x_{h}=\frac{X_{h}}{X} ; \quad(h=1, \ldots, S)
$$

when $X=0$ we can e.g. let $x_{h}=S^{-1}$.

Let $r_{k}$ denote the recoveries on the $k$ th reinstatement, that is,

$$
r_{k}=\min (\max (X-k m, 0), m) . \quad(k=0,1, \ldots, K)
$$

We also introduce the cumulative quantities

$$
R_{k}=\sum_{i=0}^{k} r_{i}=\min (X,(k+1) m) . \quad(k=0,1, \ldots, K)
$$

The total recoveries amount to $R_{K}$.

Before discussing allocation of the reinsurance premiums, we have to decide how to allocate the recoveries to the sub-portfolio. If $X \leq(K+1) m$, then there is no problem. In that case the reinsurer covers all payments to the layer, and each sub-portfolio receives its share $X_{h}$. The problem arises when $X>(K+1) m$. Then the cedant receives only $(K+1) \mathrm{m}$. We suggest that this payment should be allocated to the sub-portfolios proportional to their shares 
in $X$, that is, sub-portfolio $h$ should receive $x_{h}(K+1) m$. Thus, in general, the sub-portfolio will receive $x_{h} R_{K m}$. Analogously we suggest that the reinstatements should be allocated in the same proportion, that is, the shares of sub-portfolio $h$ in $r_{k}$ and $R_{k}$ are respectively

$$
r_{k h}=x_{h} r_{k} \quad R_{k h}=x_{h} R_{k} . \quad(k=0,1, \ldots, K ; h=1, \ldots, S)
$$

Evaluation of the moments of the $r_{k}$ 's is discussed in SUNDT (1991b). Unfortunately the situation is far more complicated for the $r_{k h}$ 's.

The discussion about the allocation of the reinsurance premiums in Sections 3-5 is also valid for other schemes for allocation of the recoveries.

\section{FREE REINSTATEMENTS}

3A. In this section we let $c_{k}=0$ for all $k$. In this case the premium $P$ is to cover $R_{K}$, and the share of sub-portfolio $h$ in this cover is $R_{K h}$. Let $P_{h}$ be the premium to be paid by sub-portfolio $h$. We should have

$$
\sum_{h=1}^{S} P_{h}=P .
$$

3B. If the premium is to be allocated by the expected value principle, we have

$$
P_{h}=(1+\alpha) \mathrm{E} R_{K h}
$$

for some loading factor $\alpha$. By summing over $h$, we obtain

$$
P=(1+\alpha) \text { E } R_{K},
$$

that is,

$$
1+\alpha=\frac{P}{\mathrm{E} R_{K}},
$$

which gives

$$
P_{h}=\frac{\mathrm{E} R_{K h}}{\mathrm{E} R_{K}} P
$$

3C. Analogously, if the premium is to be allocated by the standard deviation principle, we have

$$
P_{h}=\mathrm{E} R_{K h}+\beta \sqrt{\operatorname{Var} R_{K h}} .
$$

Thus

$$
P=\mathrm{E} R_{K}+\beta \sum_{j=1}^{S} \sqrt{\operatorname{Var} R_{K j}},
$$


which gives

$$
\beta=\frac{P \mathrm{E} R_{K}}{\sum_{j=1}^{S} \sqrt{\operatorname{Var} R_{K j}}} .
$$

Other premium calculation principles, like e.g. the variance principle, can be treated analogously.

3D. We see that with the expected value principle,

$$
p_{h}=P_{h} / P \quad(h=1, \ldots, S)
$$

was independent of $P$. This is not the case with the standard deviation principle. From (2) and (3) we obtain

$$
p_{h}=\frac{\sqrt{\operatorname{Var} R_{K h}}}{\sum_{j=1}^{S} \sqrt{\operatorname{Var} R_{K j}}}+\frac{1}{P}\left(\mathrm{E} R_{K h}-\frac{\sqrt{\operatorname{Var} R_{K h}}}{\sum_{j=1}^{S} \sqrt{\operatorname{Var} R_{K j}}} \mathrm{E} R_{K}\right)=\varphi_{h}(P) .
$$

For $P=\mathrm{E} R_{K}$, that is, no safety loading, we get the same allocation as with the expected value principle. The function $\varphi_{h}$ is monotone. Whether it is increasing or decreasing, depends on the coefficient of variation of $R_{K h}$,

$$
\psi_{h}=\frac{\sqrt{\operatorname{Var} R_{K h}}}{\mathrm{E} R_{K h}} .
$$

With

$$
\psi=\frac{\sum_{j=1}^{S} \sqrt{\operatorname{Var} R_{K j}}}{\mathrm{E} R_{K}}=\sum_{j=1}^{S} \frac{\mathrm{E} R_{K j}}{\mathrm{E} R_{K}} \psi_{j},
$$

we have

$$
\varphi_{h}(P)=\frac{\sqrt{\operatorname{Var} R_{K h}}}{\sum_{j=1}^{S} \sqrt{\operatorname{Var} R_{K j}}}+\frac{\mathrm{E} R_{K h}}{P}\left(1-\frac{\psi_{h}}{\psi}\right)
$$

From this we see that if $\psi_{h}>\psi$, then $\varphi_{h}$ is strictly increasing; if $\psi_{h}<\psi$, it is strictly decreasing; and if $\psi_{h}=\psi$, it is constant. A sub-portfolio with a high coefficient of variation would have to pay a greater share of the premiums by the standard deviation principle than by the expected value principle whereas with a low coefficient of variation it would have to pay a lower share. 
3E. Evaluation of $\mathrm{E} R_{K}, \operatorname{Var} R_{K}, \mathrm{E} R_{K h}, \operatorname{Var} R_{K h}(h=1, \ldots, S)$ becomes much easier when $K=\infty$, that is, unlimited free reinstatements. In that case

$$
R_{\infty}=\lim _{K \uparrow \infty} R_{K}=X \quad R_{\infty h}=\lim _{K \uparrow \infty} R_{K h}=x_{h} X=X_{h},
$$

and thus

$$
\begin{aligned}
& \mathrm{E} R_{\infty}=\mathrm{E} X=\mathrm{E} N \mathrm{E} Z \quad \operatorname{Var} R_{\infty}=\operatorname{Var} X=\mathrm{E} N \operatorname{Var} Z+\mathrm{E}^{2} Z \operatorname{Var} N \\
& \mathrm{E} R_{h \infty}=\mathrm{E} X_{h}=w_{h} \mathrm{E} N \mathrm{E}[Z \mid H=h] \\
& \operatorname{Var} R_{h \infty}=\operatorname{Var} X_{h}=w_{h} \mathrm{E} N \mathrm{E}\left[Z^{2} \mid H=h\right]+\left(w_{h} \mathrm{E}[Z \mid H=h]\right)^{2}(\operatorname{Var} N-\mathrm{E} N) .
\end{aligned}
$$

As it is much easier to evaluate these expressions than the ones for finite $K$, we suggest to also apply them as an approximation for finite $K$. If the subportfolios are all within the same company, it is a question whether one should bother to use more complicated allocation schemes; after all it is the same company that pays the total premium.

When $N$ is Poisson distributed, then $\operatorname{Var} N=\mathrm{E} N$, and we obtain

$$
\operatorname{Var} X=\mathrm{E} N \mathrm{E} Z^{2} \quad \operatorname{Var} X_{h}=w_{h} \mathrm{E} N \mathrm{E}\left[Z^{2} \mid H=h\right] .
$$

3F. With free reinstatements, it was relatively clear what to do when we had decided according to what premium calculation principle the allocation was to be performed, and in the special case with unlimited reinstatements the premium allocation became relatively simple. Unfortunately the situation becomes much more complicated with paid reinstatements, and even for a given premium principle, it is no longer obvious what to do. In Section 4 we shall discuss some possible allocation schemes under the expected value principle, dropping the restriction to free resintatements. In Section 5 these schemes will be adapted to the standard deviation principle.

\section{PREMIUM ALLOCATION BASED ON THE EXPECTED VALUE PRINCIPLE}

4A. We assume that sub-portfolio $h$ should initially pay the premium $P_{h A}$. Furthermore, for each reinstatement $k$ it should pay the part of the reinstatement premium that arises from recoveries allocated to that sub-portfolio, that is, $c_{k} \operatorname{Pr}_{k-1, h} / m$. As the reinstatement premiums presumably already incorporate a risk loading, we consider $P_{h A}$ separately as a premium for $r_{0 h}$ and get

$$
P_{h A}=\left(1+\alpha_{A}\right) \mathrm{E} r_{0 h}
$$

with some risk loading $\alpha_{A}$. Like in subsection $3 \mathrm{~B}$, we obtain

$$
1+\alpha_{A}=\frac{P}{\mathrm{E} r_{0}} \quad P_{h A}=\frac{\mathrm{E} r_{0 h}}{\mathrm{E} r_{0}} P
$$


Under this scheme, the initial premium $P$ should be distributed in the same proportion as the expected claims on the 0th reinstatement.

4B. The allocation scheme of subsection 4A seems reasonable if all reinstatements are at $100 \%$. On the other hand, if they are all free, it seems unnatural that the premium should be shared proportional to the pure premium of the 0th reinstatement; it would be more fair to share it proportional to the pure premium for the whole cover like suggested in Section 3. More generally, let us assume that the first $K^{\prime}$ reinstatements are free and the remaining ones paid. As a modification of the scheme of subsection $4 \mathrm{~A}$, we consider the initial premium $P$ as the premium for the initial cover and the free reinstatements, that is, the premium for $R_{K^{\prime}}$. The share of sub-portfolio $h$ in this cover is $R_{K^{\prime} h}$, and analogous to what we did in subsection $4 \mathrm{~A}$, we obtain

$$
P_{h B}=\frac{\mathrm{E} R_{K^{\prime} h} h}{\mathrm{E} R_{K^{\prime}}} P
$$

The premiums for the paid reinstatements should be allocated as described in subsection 4A.

Although we argued that free and paid reinstatements should be treated differently, this different treatment is also a bit uncomfortable. A free reinstatement is obtained as a limiting case of a paid reinstatement when letting the reinstatement percentage approach zero, and we would therefore like the premiums as functions of $c_{K^{\prime}+1}$ to be continuous at $c_{K^{\prime}+1}=0$. Unfortunately this is not the case with our present scheme. However, in practice $c_{K^{\prime}+1}$ would usually be at least $50 \%$, and thus a discontinuity at 0 is mainly of theoretical interest.

4C. It could be argued that the reinstatement premiums should be shared between the sub-portfolios in the same proportion as the initial premium, that is, if sub-portfolio $h$ should pay the initial premium $P_{h C}$, then it should pay $c_{k} P_{h C} r_{k-1} / m$ for the $k$ th reinstatement. This would to a greater extent give a risk sharing of the reinstatement premiums between the sub-portfolios. With this scheme, the total premium of sub-portfolio $h$ becomes $P_{h C} T$ with

$$
T=1+\frac{1}{m} \sum_{k=1}^{K} c_{k} r_{k-1},
$$

and this premium should cover $R_{K h}$. Unfortunately we get a similar, but smaller, problem with the expected value principle to the one we had with the standard deviation principle in Section 4 of SUNDT (1991b); these principles were not originally developed for random premium payment. However, we want the expected premium to cover the expected claim payments with a proportional loading $\alpha_{C}$, that is,

$$
P_{h C} \mathrm{E} T=\left(1+\alpha_{C}\right) \mathrm{E} R_{K h},
$$


which gives

$$
P_{h C}=\frac{\left(1+\alpha_{C}\right) \mathrm{E} R_{K h}}{E T} .
$$

As $\sum_{h=1}^{S} P_{h C}=P$, we obtain

$$
P_{h C}=\frac{\mathrm{E} R_{K h}}{\mathrm{E} R_{K}} P .
$$

With this scheme we only need to calculate $\mathrm{E} R_{K h}(h=1, \ldots, S)$. We see that we do not get the problem with distinction between free and paid reinstatements that we got with the schemes of subsections 4A-B.

If all reinstatements are free, the schemes of subsections $4 \mathrm{~B}$ and $4 \mathrm{C}$ reduce to the scheme discussed in subsection $3 B$.

4D. In subsection 3D we argued that for free reinstatements we could in some cases use the premium formula with $K=\infty$ as an approximation also when $K<\infty$. We would also suggest this as a rough approximation even with paid reinstatements. We then obtain that sub-portfolio $h$ should pay the premium

$$
P_{h D}=\frac{\mathrm{E} R_{\infty h}}{\mathrm{E} R_{\infty}}=\frac{w_{h} \mathrm{E}[Z \mid H=h]}{\mathrm{E} Z} .
$$

\section{PREMIUM ALLOCATION BASED ON THE STANDARD DEVIATION PRINCIPLE}

5A. In Section 4 we suggested four allocation schemes based on the expected value principle. We shall now adapt these schemes to the standard deviation principle.

For the scheme of subsection $4 \mathrm{~A}$ we get analogously to what we did in subsection $3 \mathrm{C}$,

$$
P_{h A}=\mathrm{E} r_{0 h}+\beta_{A} \sqrt{\operatorname{Var} r_{0 h}}
$$

with

$$
\beta_{A}=\frac{P-\mathrm{E} r_{0}}{\sum_{h=1}^{S} \sqrt{\operatorname{Var} r_{0 h}}}
$$

5B. In the same way we get for the scheme of subsection 4B

$$
P_{h B}=\mathrm{E} R_{K^{\prime} h}+\beta_{B} \sqrt{\operatorname{Var} R_{K^{\prime} h}}
$$


with

$$
\beta_{B}=\frac{P-\mathrm{E} R_{K^{\prime}}}{\sum_{h=1}^{S} \sqrt{\operatorname{Var} R_{K^{\prime} h}}} .
$$

5C. For the scheme of subsection $4 \mathrm{C}$ the situation becomes more complicated. The premium to be paid by sub-portfolio $h$ is $P_{h C} T$, and analogous to the reasoning in Section 4 of SUNDT (1991b), $P_{h C}$ should be the greatest root of the equation

$$
P_{h C} \mathrm{E} T=\mathrm{E} R_{K h}+\beta_{C} \sqrt{\operatorname{Var}\left(R_{K h}-P_{h C} T\right)}
$$

for some loading $\beta_{C}$. For given $\beta_{C}$ this is a quadratic equation in $P_{h C}$ that can easily be solved. However, $\beta_{C}$ should satisfy

$$
\sum_{h=1}^{S} P_{h C}=P
$$

and it therefore seems that we cannot solve the equation explicitly but have to find a numerical solution by iteration. By summation over $h$ in (5) we obtain

$$
P \mathrm{E} T=\mathrm{E} R_{K}+\beta_{C} \sum_{h=1}^{S} \sqrt{\operatorname{Var}\left(R_{K h}-P_{h C} T\right)},
$$

that is,

$$
\beta_{C}=\frac{P \mathrm{E} T-\mathrm{E} R_{K}}{\sum_{h=1}^{S} \sqrt{\left.\operatorname{Var} R_{K h}-P_{h C} T\right)}}
$$

A numerical solution can found iteratively by finding initial values of the $P_{h C}$ 's by e.g. (4), find a $\beta_{C}$ from (6), find new values of the $P_{h C}$ 's from (5), find a new value of $\beta_{C}$ from (6) etc., until the $P_{h C}$ 's approximately sum up to $P$.

5D. The adaption of the scheme of subsection 4D to the standard deviation principle is trivial when we apply the expressions in subsection $3 \mathrm{E}$.

\section{A SIMULATION APPROACH}

6A. Under reasonable assumptions about the relevant distributions it is relatively simple to evaluate the $P_{h D}$ 's both under the expected value principle and the standard deviation principle. However, for the other schemes, we need first order moments of the $R_{k h}$ 's for the expected value principle, and for the standard deviation principle we need the corresponding second order moments in addition. As already indicated in Section 2, analytical evaluation of these 
moments seems complicated, and we therefore suggest to estimate them by stochastic simulation. For given distributions of $N$ and $(H, Y)$ we simulate $J$ realisations of

$$
\chi=\left\{N,\left(H_{1}, Y_{1}\right),\left(H_{2}, Y_{2}\right), \ldots,\left(H_{N}, Y_{N}\right)\right\} .
$$

Each simulation is performed by first generating $N$. Then for each $i$ we first generate $H_{i}$, that is, we decide in which sub-portfolio the claim is incurred. For given $H_{i}$, we generate the claim amount $Y_{i}$ from the severity distribution of the appropriate sub-portfolio $H_{i}$. When we have found $\chi$, we construct

$$
\boldsymbol{R}=\left\{\boldsymbol{r}_{k h}\right\} \underset{k=0}{K} \underset{h=1}{S} .
$$

We estimate the relevant moments of $\boldsymbol{R}$ from the $J$ simulations by the corresponding empirical moments of the simulated values.

For the simulation scheme it is often convenient to interpret $N$ as the number of claims exceding the retention and not bother to simulate the minor claims.

6B. We have implemented this estimation scheme in the programming language Mathematica for the case when $N$ is Poisson distributed with parameter $\lambda$ and the conditional distribution of $Y$ given $H=h$ is the Pareto distribution with parameters $l$ and $\alpha_{h}$. Let $N_{h}$ be the number of claims incurred by sub-portfolio $h$, that is,

$$
N_{h}=\sum_{i=1}^{N} I\left(H_{i}=h\right) .
$$

From well-known results (cf. e.g. SundT (1991a)) we get that $N_{1}, \ldots, N_{S}$ are independent and Poisson distributed; $N_{h}$ with parameter

$$
\lambda_{h}=w_{h} \lambda \text {. }
$$

Example. We have performed a numerical study for the layer 100 xs 100 with one reinstatement at $100 \%$. There are two sub-portfolios, and we assume that $\lambda_{1}=0.02, \alpha_{1}=1.3, \lambda_{2}=0.3, \alpha_{2}=1.2$, and $P=23$. With 10000 simulations we obtain the following values of $100 P_{1} / P$.

\begin{tabular}{lcc}
\hline \hline \multirow{2}{*}{ Scheme $(t)$} & \multicolumn{3}{c}{ Principle } \\
\cline { 2 - 3 } & Expected value & Standard deviation \\
\hline$A, B$ & 94.1 & 91.1 \\
$C$ & 94.2 & 90.2 \\
$D$ & 93.9 & 92.5 \\
\hline
\end{tabular}


The values for the standard deviation principle are in general lower than the corresponding values for the expected value principle; the relative fluctuations are lower in sub-portfolio 1 than in sub-portfolio 2 .

\section{GENERALISATIONS}

7A. In Section 2 we assumed that each claim totally falls within one sub-portfolio. However, in practice one claim event often affects more than one sub-portfolio. For instance, a hurricane could hit several geographical areas and several types of insurance (accident, building, automobile, marine, etc.). If the treaty is per event, such an event could produce a claim that should be shared between the sub-portfolios. To be able to handle such situations we have to generalise our model.

Let $\boldsymbol{Y}_{i}=\left(Y_{i 1}, \ldots, Y_{i S}\right)^{\prime}$ with $Y_{i h}$ denoting the part of claim $i$ that falls within sub-portfolio $h$. We have $Y_{i}=\sum_{h=1}^{S} Y_{i h}$. In the special case introduced in Section 2 we had $Y_{i h}=I\left(H_{i}=h\right) Y_{i}$. We assume that the $Y_{i}^{\prime}$ 's are mutually independent and identically distributed and independent of $N$.

Unfortunately, when a claim can affect more than one sub-portfolio, we do not only have the problem of how to allocate the premium, but also how to allocate the claims to the layer. A simple solution is to define the share of sub-portfolio $h$ in claim $i$ to the layer as

$$
Z_{i h}=\frac{Z_{i}}{Y_{i}} Y_{i h},
$$

that is, $Z_{i}$ is shared between the sub-portfolios proportionally to their shares in $Y_{i}$.

7B. Another generalisation is to introduce a dependence between the number of claims and the individual claim amounts. Without regarding sub-portfolios, such models are discussed by SUNDT (1991c).

\section{ACKNOWLEDGEMENT}

The present research was performed while the author worked with Uni Storebrand International Insurance A/S. The author is grateful to an anonymous referee for suggesting the definition of the $r_{k h}$ 's given in Section 2 .

\section{REFERENCES}

SundT, B. (1991a) An introduction to non-life insurance mathematics. (2. ed.) Verlag Versicherungswirtschaft, Karlsruhe.

SundT, B. (1991b) On excess of loss reinsurance with reinstatements. Bulletin of the Swiss Association of Actuaries, 51-66.

SundT, B. (1991c) On approximating aggregate claims distributions and stop-loss premiums by truncation. Insurance: Mathematics and Economics 10, 133-136.

\section{BJøRN SUNDT}

Grønnegaten 5, N-0350 Oslo 3, Norway. 